\title{
Erratum: Correlation energy of the uniform electron gas from an interpolation between high- and low-density limits [Phys. Rev. B 81, 085123 (2010)]
}

Jianwei Sun, John P. Perdew, and Michael Seidl

(Received 4 January 2018; published 14 August 2018)

DOI: 10.1103/PhysRevB.98.079903

Equation (A3) propagates a transcription error from Ref. [1]. In this equation, $\tan ^{-1}(u)$ should be replaced by $\tan ^{-1}(1 / u)$. A detailed derivation is presented in Ref. [2]. The parametrization of $a_{1}(\varsigma)$ in Table II, and its $|\varsigma| \rightarrow 1$ limit are not accurate. The error in this limit was pointed out in Ref. [3]. However, the correction has only a minor effect on the density parameter interpolation (DPI) and no effect in the spin-unpolarized limit. The corrected parametrization and corrected DPI are presented in Ref. [2].

[1] J. P. Perdew and Y. Wang, Phys. Rev. B 45, 13244 (1992).

[2] P. Bhattarai, A. Patra, C. Shahi, and J. P. Perdew, Phys. Rev. B. 97, 195128 (2018).

[3] P.-F. Loos and P. M. W. Gill, Phys. Rev. B 84, 033103 (2011). 